\title{
Investing and Price Competition for Multiple Bands of Unlicensed Spectrum
}

\author{
Chang Liu \\ EECS Department \\ Northwestern University, Evanston, IL 60208 \\ Email: changliu2012@u.northwestern.edu
}

\author{
Randall A. Berry \\ EECS Department \\ Northwestern University, Evanston, IL 60208 \\ Email: rberry@eecs.northwestern.edu
}

\begin{abstract}
Motivated in part by the success of WiFi, there has been much interest in opening up new "prime" bands of spectrum for unlicensed use. Such bands can lower the cost for new wireless service providers to enter a market. The increased competition could in turn improve economic welfare. However, the openness of such spectrum can also lead to it becoming over congested, which in turn could deter investment. Indeed, a recent paper shows that due to the risk of congestion, only a single service provider may invest in a given unlicensed band and charge monopoly prices. However, that paper considers only a single band of unlicensed spectrum. In this paper, we consider investment and competition when there are multiple unlicensed bands available. In such a setting, each service provider could either invest in a single band or spread its investment over multiple bands. We consider a two-stage game for such a setting, in which firms first decide investment levels and second compete for customers by announcing prices for their service. The equilibria and resulting welfare of this game are characterized.
\end{abstract}

\section{INTRODUCTION}

Due in part to the scarcity of wireless spectrum and the success of $\mathrm{WiFi}$, there is much interest in expanding the amount of unlicensed spectrum. In particular, there has been interest in opening up "prime" spectrum with better propagation characteristics for unlicensed access, which would enable unlicensed providers to serve a larger number of customers per access point. Indeed, the TV white spaces in the U.S. are an example of one such band that is now available for unlicensed use (though only in areas where incumbent TV stations are not present) [1].

A leading argument for unlicensed spectrum is that not requiring expensive licenses will lower the barrier to entry and thus increase competition, benefiting consumers and the overall economy. However, though licenses are not required, firms still must invest in infrastructure to offer services. The openness of an unlicensed band can also lead to the band becoming over congested if too many firms invest. However, the risk of this occurring could also deter firms from investing or instead could motivate them to invest in better technology. The goal of the paper is to study such issues when there are multiple bands of unlicensed spectrum available for firms to invest in.

Our approach in this paper is based on the model in [3] and [4], which study models for investment and price competition

This research is supported in part by NSF grants CNS-1147786, SES1247984 and SES-1343381. in a single band of unlicensed spectrum. This in turn was based on [2], which adapted models of competition with congestible resources from the economics literature and operations literature (e.g., [5], [6]) to study price competition with a single band of unlicensed spectrum, assuming all investment was sunk. Related models have also been applied in [6], [7] to study price competition with shared spectrum.

In [3] and [4], investment and price competition was modeled as a two-stage game among a set of wireless service providers (SPs). The SPs first simultaneously make investment decisions and then compete for customers by announcing service prices. The main result of [3] and [4] is that with a single band of unlicensed spectrum in many cases the only (pure strategy) equilibria of this game is for only one SP to invest and to charge monopoly prices. In other words, making a band open does not necessarily lead to greater competition.

In this paper, we generalize [3] and [4] by considering models where there are multiple bands of spectrum available and a service provider must decide how much to invest in each band as well as what price to charge for service in that band. This is of interest both because in practice there are multiple bands of unlicensed spectrum available and having such bands may allow multiple providers to enter the market, for example by different providers deciding to invest in different bands. Indeed, our analysis will show that such equilibria are possible. However, we will also show that there may also still be equilibria in which a single monopolist emerges.

The rest of the paper is organized as follows. Our model is described in Section II. We then begin by studying the investment and pricing decision that a monopolist would make given two bands of spectrum. Following this we consider the problem when there are multiple competing SPs in Sections IV and V. Finally, we conclude in Section VI.

\section{MOdeL}

\section{A. A Two-stage Game}

As in [3], we consider a two-stage investment and price competition game where SPs first invest in their service and then compete for the same pool of customers by announcing prices. For most of the paper we assume that there are $N$ SPs in the market who may invest in two bands of unlicensed spectrum, denoted by $A$ and $B$, though most of the results 
can be generalized to more than two bands. The SPs simultaneously invest in the first stage, where $I_{i}^{A}$ and $I_{i}^{B}$ denote the investment by SP $i$ in band $A$ and $B$, respectively. In particular, each SP $i$ can choose to not invest at all, to invest in only one of the two bands, or to spread their invest across both bands. After making their investment decisions, each SP $i$ announces prices, $p_{i}^{A}$ and $p_{i}^{B}$, respectively, for their service in band $A$ and band $B$. Note we allow the SPs to announce different prices in each band and thus view each band as offering a distinct service. For each SP $i$, its ultimate goal is to maximize its total profit, $f_{i}$, which is the difference between revenue and expenses across the two bands, i.e.,

$$
f_{i}=\sum_{K \in\{A, B\}} f_{i}^{K}=\sum_{K \in\{A, B\}} p_{i}^{K} x_{i}^{K}-c I_{i}^{K} .
$$

Here, $x_{i}^{K}$ is the number of customers the $i$ th SP serves in band $K$ (where $K \in\{A, B\}$ ). We call a SP is active in band $K$ if $I_{i}^{K}>0$. If an SP is inactive in band $K$, i.e., $I_{i}^{K}=0$, then its profit in band $K$ is zero.

1) Supply: As in [3] customers select bands based on not only the announced prices but on the quality of service they can obtain in each band. We model this via a congestion cost for the customers of SP $i$ in band $K$, which is given by $g\left(x^{K}, I_{i}^{K}, W^{K}\right)$, where $x^{K}=\sum_{i} x_{i}^{K}$, is the sum of $x_{i}^{K}$, the customers served in band $K$ by SP $i$, and $W^{K}$ is the bandwidth of that band. In general $g$ will be increasing in $x^{K}$, the total traffic of all SPs, reflecting the fact that the unlicensed band is shared by all SPs. Further we assume that $g$ is decreasing in $I_{i}^{k}$ and $W^{K}$. Hence, for each SP, more investment lowers the congestion its customers experience thus allowing it to charge higher prices or serve more customers. For this paper we consider the specific example where $g\left(x^{K}, I_{i}^{K}, W^{K}\right)=$ $g\left(\frac{x^{K}}{I^{K} W^{k}}\right)$. An example of this for a single band is shown in Fig. 1. Further, for the case where the two bands have the same bandwidth, we set $W^{K}=1$ to simplify our notation. ${ }^{1}$

2) Demand and Equilibria: Customers select a single SP and band from which to receive service (or choose not to be served). Given the announced prices, $\mathbf{p}=$ $\left(p_{1}^{A}, p_{2}^{A}, \ldots, p_{N}^{A}, p_{1}^{B}, p_{2}^{B}, \ldots, p_{N}^{B}\right)$, and congestion costs based on investment, $\mathbf{I}=\left(I_{1}^{A}, I_{2}^{A}, \ldots, I_{N}^{A}, I_{1}^{B}, I_{2}^{B}, \ldots, I_{N}^{B}\right)$, from all SPs, each customer chooses the one SP in one band with the smallest delivered price, which is the sum of announced price and congestion cost each customer experiences from that SP in that band. Hence, for SP $i$, its delivered price in band $K$ is given by $p_{i}^{K}+g\left(x^{K}, I_{i}^{K}, W^{K}\right)$ (again see Fig. 1 ).

We assume a unit mass of customers whose demand is given by a downward sloping function $D(p)$ with inverse function $P(q)$. That is up to a mass of $q$ customers are willing to pay delivered price $P(q)$ for the service. Generally we assume $P(q)$ is concave and decreasing. Each customer is infinitesimal so that a single customer has a negligible effect on the congestion in any band. Hence, given the investment and price of every SP, $(\mathbf{I}, \mathbf{p})$, the demand vector $\mathbf{x}=\left(x^{A}, x^{B}\right)$, is assumed to

\footnotetext{
${ }^{1}$ It can be seen that from the following that this assumption is without loss of generality if we appropriately adjust the investment cost $c$ of each SP.
}

satisfy the following Wardrop equilibrium conditions [9]:

$$
\begin{gathered}
p_{i}^{K}+g_{i}\left(x^{K}\right)=\min _{i \in N}\left(p_{i}^{K}+g_{i}\left(x^{K}\right)\right), \text { for } i \in N \text { with } x_{i}^{K}>0, \\
p_{i}^{K}+g_{i}\left(x^{K}\right)=P(X), \text { for } i \in N \text { with } x_{i}^{K}>0, \\
p_{i}^{K}+g_{i}\left(x^{K}\right) \geq P(X), \forall i \in N .
\end{gathered}
$$

Here $X=\sum_{K \in A, B} x^{K}$ and $g_{i}\left(x^{K}\right)=g\left(x^{K}, I_{i}^{K}, W^{k}\right)$. These conditions specify that the delivered price of all SPs serving customers are equal and no greater than $P(X)$. A (Nash) equilibrium of the overall game is one in which the customers are in a Wardrop equilibrium and no SP can improve their profit by changing either their investment or price. ${ }^{2}$ Further, to ensure that equilibria exist we make the assumption that if one SP announces a zero price in a band for which another SP is announcing a strictly positive price, then the former SP will serve zero customers.

At an equilibrium, the welfare obtained by the $x$ th customer is the difference between the delivered price it pays and the amount it is willing to pay, given by $P(x)$. The total consumer welfare is the integral of this over all customers. The total welfare of the market is the sum of the consumer welfare and the SPs' profits.

\section{B. Investment in a Single Band}

The next lemma summarizes some results from [3] for the same investment and pricing game with a single unlicensed band and congestion cost $g\left(\frac{x}{I_{i}}\right)$.

Lemma 2.1: Given two SPs competing in a single band with investment vector $\left(I_{1}, I_{2}\right)$ and price vector $\left(p_{1}, p_{2}\right)$, in equilibrium, the following conditions hold: (i) If $I_{1}=I_{2}$, then $p_{1}=0, x_{1}>0, p_{2}=0$ and $x_{2}>0$. (ii) If $I_{1}>I_{2}>0$, then $p_{1}>0, x_{1}>0$ and $p_{2}=0, x_{2}=0$.

This lemma shows that the only profitable way for a SP to invest and offer service is if it invests more than its competitor. Indeed, it follows that if an equilibrium exists in the overall game, then it must be one in which a single SP invests and acts as a monopolist. ${ }^{3}$

Here, we differ from [3] in that there are two bands of spectrum available and each SP can invest in one or both bands. Assume there are two SPs in the market, then it follows directly from Lemma. 2.1 that if both SPs invest in the same band, then only the one with the larger investment can make a profit. Hence, such an investment profile will never exist in equilibrium. However, now the two SPs can each invest in a separate band, in which case this lemma does not apply.

\section{Discussion}

Before continuing we briefly discuss some of the assumptions in our model. No fixed cost was associated with investment, i.e., there is only the variable costs associating with improving the service quality of an SP's customers. Adding a

\footnotetext{
${ }^{2}$ More precisely we consider subgame perfect Nash equilibria so that for any fixed investment profile, the SPs all charge prices which are an equilibrium of the corresponding pricing sub-game.

${ }^{3}$ Under some additional conditions given in [7] it is shown that such an equilibrium does exist.
} 


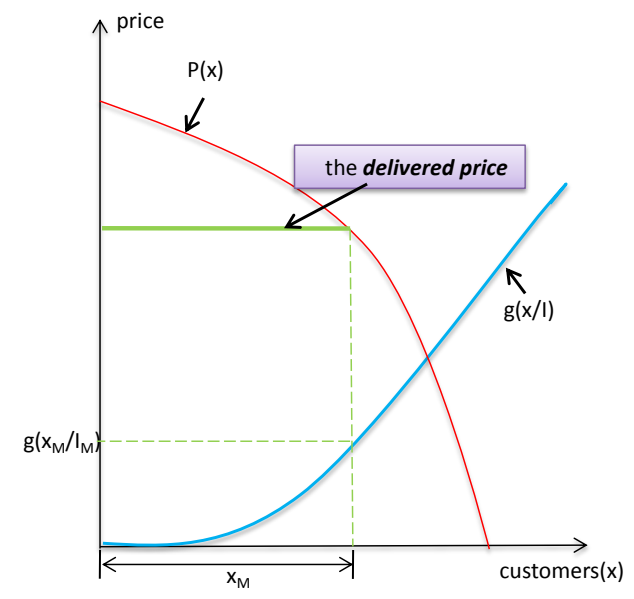

Fig. 1. Illustration of two-stage investment game with single SP and single band.

fixed cost per band, could incentivize providers to only invest in one band (to reduce their fixed costs). We did not adopt such a model as we wanted to isolate how the competition among providers affected each SP's investments. In addition, we did not model any economies of scale that a provider might have either within a given band or across bands, i.e., the marginal cost for investment is constant. Again this enables us to focus on how competition affects a SP's investment decisions in a simple setting.

The congestion cost we consider, i.e., $g\left(\frac{x^{k}}{I_{i}^{k} W^{k}}\right)$, is one where each SP's investments reduce the congestion seen by their own customers, but do not effect the congestion of other SPs' customers; for example this could model an uplink scenario where a SP invests in adding sectors at its access points. As discussed in [4], there are several other ways that investment could be modeled in terms of how investment decisions affect the congestion costs for users of each SP. The results we obtain from this paper also hold for some of these other modes, but the details are omitted due to space considerations.

\section{Monopoly Investment With Two Bands of SPECTRUM}

In this section, we consider a monopoly scenario, i.e., only one SP exists in the market. Given two bands available, the monopolist can choose to invest solely in either band or in both. To maximize its profit, it solves:

$$
\begin{array}{ll}
\max & p^{A} x^{A}+p^{B} x^{B}-c\left(I^{A}+I^{B}\right) \\
\text { subject to } & p^{A}+g\left(x^{A} /\left(I^{A} W^{A}\right)\right)=P\left(x^{A}+x^{B}\right), \\
& p^{B}+g\left(x^{B} /\left(I^{B} W^{B}\right)\right)=P\left(x^{A}+x^{B}\right), \\
& 0 \leq x^{A}, x^{B} \leq 1, \\
& I^{A}, I^{B} \geq 0 .
\end{array}
$$

This problem can be re-written so that if the monopolist uses a given band, its investment decision in that band is simply to maximize the term $\Delta(x /(I W))=g(x /(I W))+$ $(c / W)((I W) / x)$. Let $t=x /(I W)$, then $\Delta$ can be re-written as

$$
\Delta(t)=g(t)+c /(W t) .
$$

We can then view the monopolist as optimizing over the prices (or equivalently the $x$ values) and the $t$-values for each band. Further, if it invests in a given band then the optimization over $t$ is simply given by solving $\Delta^{*}=\min _{t \geq 0} \Delta(t)$, which is a convex problem for our model. The solution to this problem depends on the bandwidth $W$. Next we consider two cases for these parameters: one where they are the same for each band and one where they are different.

\section{A. Two Homogeneous Bands}

If the two bands have the same bandwidth, i.e., $W^{A}=$ $W^{B}=1$, then if the SP invests in both bands, each band will have the same value of $\Delta^{*}$. Hence, its profit optimization can be written as :

$$
\begin{array}{ll}
\max & \left(P\left(x^{A}+x^{B}\right)-\Delta^{*}\right)\left(x^{A}+x^{B}\right) \\
\text { subject to } & 0 \leq x^{A}, x^{B} \text { and } x^{A}+x^{B} \leq 1 .
\end{array}
$$

Letting $x=x^{A}+x^{B}$, then it can be seen that this problem is equivalent to solving

$$
\begin{array}{ll}
\max & \left(P(x)-\Delta^{*}\right) x \\
\text { subject to } & 0 \leq x \leq 1 .
\end{array}
$$

This problem has a strictly convex objective function and a convex, compact constraint set and so has a unique solution, given by solving the equation $P(x)^{\prime} x+P(x)=\Delta^{*}\left(t^{*}\right)$, where $x$ is the total customers served. Then the investment can be found by solving $x^{A} / I^{A}=x^{B} / I^{B}=t^{*}$ and $x^{A}+x^{B}=x$. Note that these equations have multiple solutions. For example, the SP could divide the traffic evenly between the two bands, yielding $I^{A}=I^{B}=x / 2 t^{*}$. If the SP invests in only one band, e.g., band A, then its optimization is again given by (1), where now $x$ denotes the traffic served in the one band the SP invested in.

Clearly this has the same solution as in the two bands case. ${ }^{4}$ An illustration of these two equilibriums is shown in Fig. 2. Moreover, since the total customers served are the same, it follows that consumer welfare and social welfare are identical as well, as summarized next.

Lemma 3.1: With two homogeneous bands, a monopolist is indifferent between investing in one band or investing in both bands; moreover, both result in the same consumer welfare and social welfare.

\section{B. With Two Heterogeneous bands}

In this section we assume that $W^{A}>W^{B}$. For each band $K$, let

$$
\Delta_{K}(t)=g(t)+c /\left(W^{K} t\right)
$$

\footnotetext{
${ }^{4}$ One might wonder why the single-band result does not follow directly from the multi-band result by letting $x_{B}$ and $I_{B}$ go to zero. However not that the reformulation using $\Delta$ only applies for $I_{B}>0$ and so this limiting case needs to be treated separately.
} 


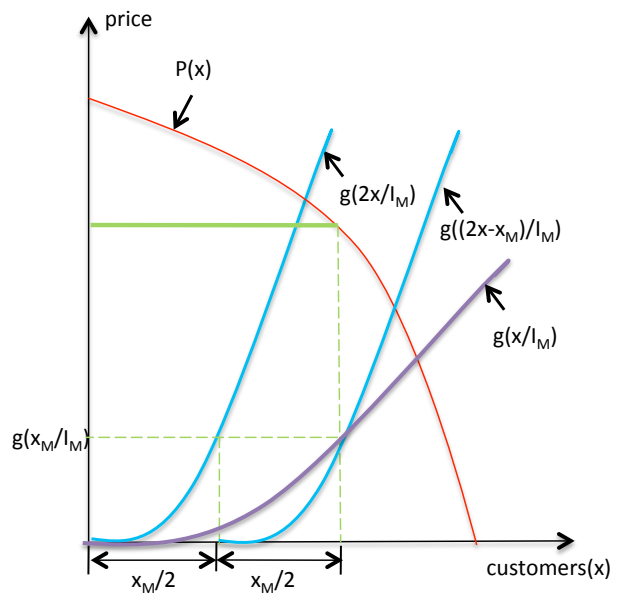

Fig. 2. Illustration of the investment game with single SP and two bands.

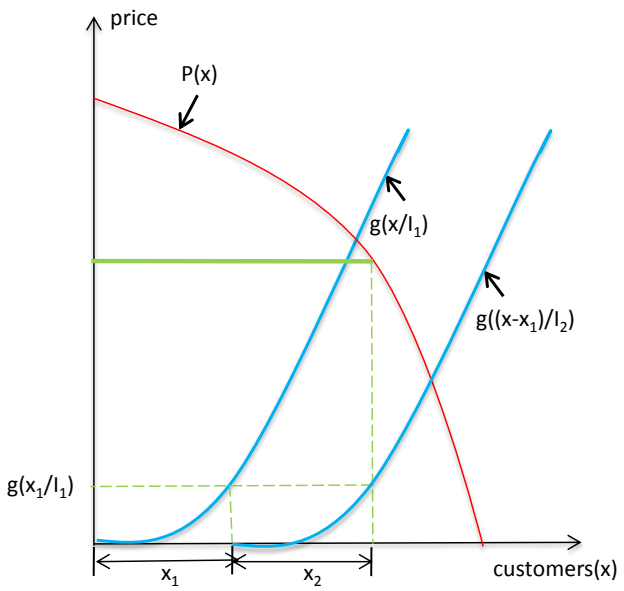

Fig. 3. Equilibrium of two SPs investing in one separate band.

and let $\Delta_{K}^{*}=\min _{t \geq 0} \Delta_{K}(t)$, which as before gives the monopolist's optimal investment if it uses band $K$. Since $\Delta_{K}(t)$ is convex, the solution to this minimization is given by the first order optimality condition: $g^{\prime}(t) t^{2}=c / W^{K}$. From this it follows that the minimum value $t_{K}^{*}$ is decreasing in $W^{k}$. It follows that

$$
\Delta_{K}^{*}=g\left(t_{K}^{*}\right)+g^{\prime}\left(t_{K}^{*}\right) t .
$$

As $g(t)$ is convex and increasing in $t, \Delta_{K}^{*}\left(t^{*}\right)$ is also an increasing function of $t^{*}$. Using the fact that $t_{K}^{*}$ decreases with $W_{K}$, it follows that the band with larger value of $W_{K}$ will always yield a smaller value of $\Delta_{K}^{*}$ and thus the monopolist will always obtain a larger profit by investing in that band over investing in the other band. Similarly, this will always be more profitable than investing in both bands.

Lemma 3.2: With two heterogeneous bands, a monopolist will only invest in the band having the larger bandwidth.

\section{Duopoly Investment Game}

In this section, we consider a scenario with two SPs and two identical bands. From Lemma. 2.1, we know that with a single band only one SP can be active in any equilibrium. This is true for two-bands as well. With this restriction there are two possible types of equilibria. In one type, each SP invests in a different band and the two SPs compete. In another type of equilibrium, a single SP invests in both bands and acts like a monopolist. The next theorem shows that both types of equilibria may exist.

Theorem 4.1: In a duopoly investment game with two homogeneous bands, there are only two types of equilibria that may exist: one where each SP invests in a different band and one where a single SP invests in both bands. The first type of equilibrium always exists; a sufficient condition for the second type of equilibrium to exist is if

$$
P\left(x^{*}\right) \leq \Delta^{*},
$$

where $x^{*}$ is given by finding the value of $x$ such that $P(x)=$ $g\left(x / I_{M}\right)$ with $I_{M}$ being the monopolist investment level.

Proof: We begin with the existence of the first type of equilibrium in Theorem. 4.1. In equilibrium, suppose SP 1 invests $I_{1}^{A}$ in band $A$ and announces price $p_{1}^{A}$ serving $x_{1}$ customers with profit $f_{1}$ while SP 2 invests $I_{2}^{B}$ in band $B$ and announces price $p_{2}^{B}$ serving $x_{2}$ customers. To show this is an equilibrium, consider a deviation in investment by SP 1, with SP 2's investment strategy fixed. Suppose that SP 1 deviates by investing a total amount $I_{1}$, which is divided into $\alpha I_{1}$ and $\beta I_{1}$ for band $A$ and $B$ respectively, where $\alpha+\beta=1$. Suppose after doing this SP 1 serves $x_{1}^{A}$ and $x_{1}^{B}$ customers in equilibrium (for the pricing subgame), with $x_{1}^{A}+x_{1}^{B}=\tilde{x}_{1}$. Then the new firm profit, $f_{1}$, for SP 1 is given by

$$
\begin{aligned}
\tilde{f}_{1}= & P\left(x_{1}^{A}+x_{1}^{B}+\tilde{x}_{2}\right)\left(x_{1}^{A}+x_{1}^{B}\right)-\left[g\left(\frac{x_{1}^{A}}{\alpha I_{1}}\right) x_{1}^{A}\right. \\
& \left.+g\left(\frac{x_{1}^{B}+\tilde{x}_{2}}{\beta I_{1}}\right) x_{1}^{B}\right]-(\alpha+\beta) c I_{1} .
\end{aligned}
$$

Here, $\tilde{x}_{2}$ is the traffic SP 2 serves in the new equilibrium of the pricing sub-game. As in equilibrium, $x_{1}>0$, thus we have

$$
\begin{aligned}
\tilde{f}_{1} & <P\left(\tilde{x}_{1}+\tilde{x}_{2}\right) \tilde{x}_{1}-\left[g\left(\frac{x_{1}^{A}}{\alpha I_{1}}\right) x_{1}^{A}+g\left(\frac{x_{1}^{B}}{\beta I_{1}}\right) x_{1}^{B}\right]-c I_{1} \\
& \leq P\left(\tilde{x}_{1}+\tilde{x}_{2}\right) \tilde{x}_{1}-g\left(\frac{x_{1}^{A}+x_{1}^{B}}{(\alpha+\beta) I_{1}}\right) \tilde{x}_{1}-c I_{1} \\
& =P\left(\tilde{x}_{1}+\tilde{x}_{2}\right) \tilde{x}_{1}-g\left(\frac{\tilde{x}_{1}}{I_{1}}\right) \tilde{x}_{1}-c I_{1} \\
& \leq f_{1} .
\end{aligned}
$$

The first inequality follows since dropping SP 2's traffic only reduces the congestion cost, the second inequality is shown in the appendix, and the final inequality follows since for $f_{1}$ to be an equilibrium it must be that it maximizes SP 1's profit given that it invests only in band $A$ (given SP 2's strategy is fixed).

The above shows that SP 1 will have no incentive to shift its investment from the conjectured equilibrium. The same goes for SP 2. Thus each SP investing in one separate band is an equilibrium as shown in Fig. 3. 
Next we turn to the second type of equilibrium where only one SP, e.g., SP 1, is active and invests in both bands, for example by investing $I_{M} / 2$ and serving $x_{M} / 2$ customers in each band. If this was not an equilibrium then SP 2 would want to enter the market and compete with SP 1 in one (or both) of the bands). From Lemma 2.1 it follows that SP 2 would need to invest more than SP 1 in one band (i.e., more than $I_{M} / 2$ ). Once, it does this, since SP 1's investment is sunk, the resulting price competition would drive SP 1's price to zero, meaning that the delivered price can be no more than $P\left(x^{*}\right)$ as defined in this theorem. Furthermore, SP 2's revenue for each user served can not be more than the difference between the delivered price and $\Delta^{*}$, where $\Delta^{*}$ is calculated as in the monopoly case. It follows that if $\Delta^{*}>P\left(x^{*}\right)$ such a deviation will not be profitable and SP 2 would not enter the market.

The next result shows that when the congestion cost is linear, i.e., $g(x / I)=x / I$, both types of equilibria exist.

Theorem 4.2: In a duopoly investment game with two homogeneous bands, if the congestion is linear, then both types of equilibria always exist.

The proof of this is shown in the Appendix.

An example of the firms' profits, customer welfare and social welfare for both types of equilibriums are shown in Fig. 5, where both congestion cost and inverse demand are linear, i.e., $g(x / I)=x / I$ and $P(x)=1-x$. As the marginal investment cost $c$ grows, all of these welfare measures decrease for both equilibria. The corresponding equilibria investment levels, customers served and prices are shown in Fig. 6. In the monopoly case, when $c$ is zero, the monopolist will invest as much as possible to get the maximum profit which is capped at 0.25 due to assumed demand curve (and the fact that only a single price is used). However, in the duopoly case, when $c$ is zero, i.e., there is no investment cost, each SP's investment are interestingly capped by 1.3 and the total profit gained is capped by 0.15 which is significantly lower than the monopoly case. However, this drop does not benefit the customers when $c=0$, i.e., both equilibria have the same consumer welfare. What is happening in the duopoly case is that the threat of competition prevents the the SPs from investing too much even though there is no cost for investment. In particular it can be seen that if the SPs invested an arbitrarily high amount, this would be then become a classic Bertrand model and neither provider would get any profit. In Fig. 5, although the duopoly case provides more consumer welfare when the marginal investment cost is small (but not zero), the consumer welfare for the monopoly case goes above the duopoly case when the investment cost becomes large. Also in Fig. 6, with large marginal cost $c$, the monopolist will serve more customers than in the duopoly scenario while asking a price close to $1 / 2$. However, the price goes to zero for the duopoly case.

When two service providers invest in two heterogeneous bands with different bandwidths, the equilibria will highly depend on the available bandwidths in both bands and the corresponding marginal investment cost for SPs, $c$. For instance, given linear demand and congestion costs as $P(x)=1-x$

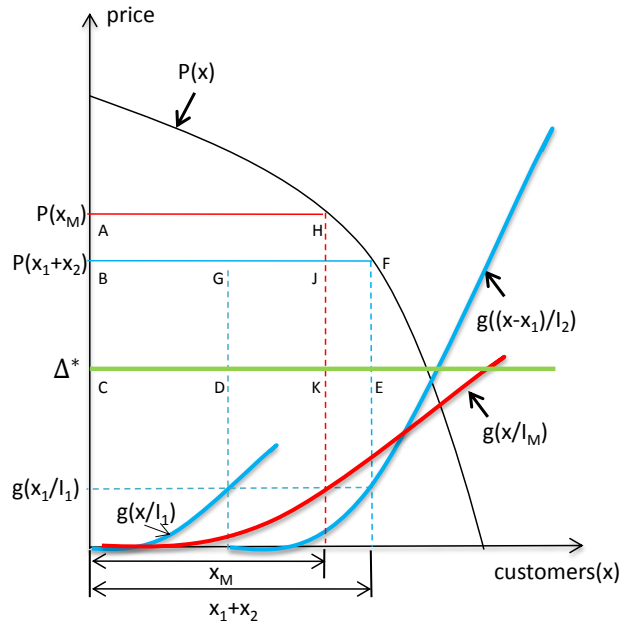

Fig. 4. Comparison of the two-stage game in case of monopoly and duopoly.

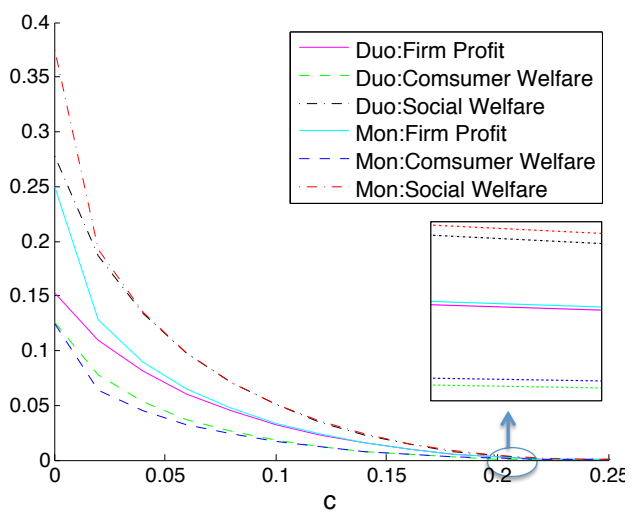

Fig. 5. Welfare analysis in duopoly game w.r.t. investment marginal cost $c$ where $B_{1}=B_{2}=1$.

and $g(x / I)=x /\left(W^{K} I\right)$, with two different bandwidths, $W^{A}$ and $W^{B}$, there is no investment when both the marginal cost and bandwidth ratio exceeds 1 , i.e., $c / W^{A}>1$ and $c / W^{B}>1$. If both ratios are under $1 / 4$, then there exists an equilibrium where both service providers invest in separate bands. However, when one band has large bandwidth and marginal cost ratio while the ratio for the other one is small, i.e., $c / W^{A}<1$ and $c / W^{B}>1$, only one service provider will invest in band $A$ and the other service provider will not invest, leaving band $B$ empty.

\section{Multiple SPS AND SPeCtrum bands}

In this section, we consider a setting when there are $n$ identical bands available with $n$ SPs. Extending our previous results, there exists one equilibrium where each SP invests in one band where no two SPs invest in the same band. In that case, each SP $i(i=1,2, \ldots, \mathrm{n})$ solves the following optimization problem:

$$
\begin{array}{ll}
\max & p_{i} x_{i}-c I_{i} \\
\text { subject to } & p_{i}+g\left(x_{i} / I_{i}\right)=P\left(x_{1}+x_{2}+\ldots+x_{n}\right) \\
& 0 \leq x_{i} \leq 1, I_{i} \geq 0, \forall i
\end{array}
$$




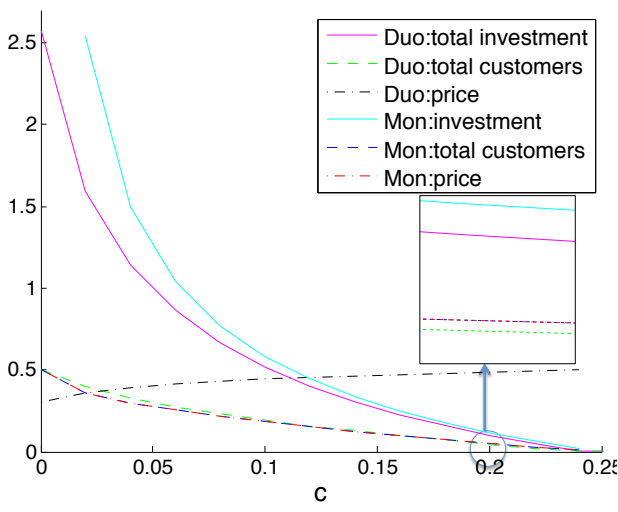

Fig. 6. Comparison of the two NEs in duopoly game w.r.t. investment marginal cost $c$.

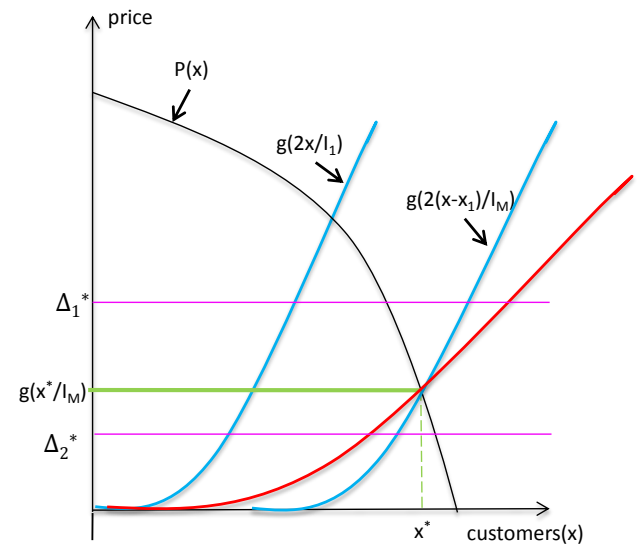

Fig. 7. Illustration of an equilibrium with multiple bands.

Again the number of customers each SP serves satisfies

$$
P\left(x_{1}+x_{2}+\ldots+x_{n}\right)+P^{\prime}\left(x_{1}+x_{2}+\ldots+x_{n}\right) x_{i}=\Delta^{*} .
$$

This equality holds for any SP which shows that all of them serve an equal number of customers, i.e, $x_{1}=x_{2}=\ldots=x_{n}$. For each SP, the firm profit can be written as $f_{i}=\left(P\left(x_{1}+\right.\right.$ $\left.\left.x_{2}+\ldots+x_{n}\right)-\Delta^{*}\right) x_{i}=-P\left(n x_{i}\right) x_{i}^{2}$, which decreases with $n$. Thus although more bands are available, each firm gains less profit with more SPs active in the market. We summarize the result in the following lemma.

Lemma 5.1: When $n$ SPs are active with $n$ identical bands of spectrum, each SP's profit decreases with $n$.

Similar to the two-band case in the previous section, there can exist another equilibrium where only one SP is active across all bands, and in fact there can be other equilibria where some SP's are active on disjoint subsets of the bands, and others are inactive. For two symmetric bands, we showed that, a sufficient condition for such an equilibrium to exist is that for any active band $P\left(x^{*}\right) \leq \Delta^{*}$, which ensures that no other SPs have an incentive to enter the market. This condition holds here as well as shown below. ${ }^{5}$

Lemma 5.2: With $n$ symmetric bands of spectrum, there exists an equilibrium where only one firm enters the market if the following condition holds:

$$
P\left(x^{*}\right) \leq \Delta^{*}
$$

where $x^{*}$ satisfies $P\left(x^{*}\right)=g\left(x^{*} / I_{M}\right)$ and $\Delta^{*}=$ $\min _{t} g(t)+c / t$.

This can be derived the same way as in the two-band case. Note that the condition in the Lemma does not depend on $n$. This is because for any $n$ an optimal investment of a monopolist that uses $n$ bands is to invest equally in all $n$ bands and serve the same traffic in each. For the given congestion cost, the monopolist can make the same profit by investing $n$ times that amount in one band and serving all the traffic in that band. Hence, the amount a monopolist will invest does not vary with the number of bands $n$ and neither does the minimum delivered price it can offer given that investment, which is $P\left(x^{*}\right)$.

\section{CONCLUSIONS}

In this paper, we analyzed a two-stage investment and pricing game with multiple bands of unlicensed spectrum. Given two identical bands, we showed that a monopolist would be indifferent between investing in one band or both. When faced with competition, two different types of equilibria can emerge - one in which different SPs invest in different bands and one in which a single SP invests in both bands and acts as a monopolist. The former generally yields higher consumer welfare, but smaller firm profits and overall welfare, though interestingly for large investment costs the monopolist equilibrium may yield higher consumer welfare.

One direction for future work is to consider different models for how investment effects congestion costs as in [4]. Another direction is to consider multiple rounds of investment (as a repeated game). In this case new strategic interactions are possible, such as a firms over investing in one stage to prevent another firm from entering at a later stage.

\section{REFERENCES}

[1] Federal Communications Commission, "Unlicensed operation in the TV broadcast bands ; additional spectrum for unlicensed devices below 900 $\mathrm{MHz}$ and in the $3 \mathrm{GHz}$ band" second memorandum opinion and order, 2010.

[2] T. Nguyen, H. Zhou. R. Berry, M. Honig, and R. Vohra, "The impact of additional unlicensed spectrum on wireless services competition," 2012 IEEE DYSPAN, pp. 146-155, May 2011.

[3] H. Zhou, R. Berry, M. Honig, R. Vohra, "Investment and Competition in Unlicensed Spectrum", 46th Annual Conference on Information Sciences and Systems (CISS), 2012.

[4] T. Nguyen, H. Zhou, R. Berry, M. Honig, R. Vohra, "The Impact of Investment Models on Service Competition in Unlicensed Spectrum", IEEE Workshop on Smart Data Pricing, 2014.

[5] D. Acemoglu, A. Ozdaglar, "Competition and Efficiency in Congested Markets," Mathematics of Operations Research., Feb. 2007

\footnotetext{
${ }^{5}$ We state this for the case where a single firm enters and acts as a monopolist; a similar result holds for the case were a subset of the firms enter, except in that case $I_{M}$ must be replaced by the optimal investment of the firms that entered the market.
} 
[6] R. Johari, G. Y. Weintraub, B. V. Roy, "Investment and Market Structure in Industries with Congestion," Operations Research., Sep. 2010

[7] C. Liu and R. Berry, "Competition with Shared Spectrum," 2014 IEEE DySPAN, Apr. 2014.

[8] C. Liu, R. A. Berry, "Competition with Licensed Shared Spectrum", Proceedings of 48th Annual Conference on Information Sciences and Systems, Mar. 2014.

[9] J. G. Wardrop, "Some theoretical aspects of road traffic research," Proceedings, Institute of Civil Engineers, PART II, vol. 1, pp. 325378, 1952.

\section{APPENDIX}

\section{A. Proof for Section III-B}

In Sec. III-B, to maximize the firm profit, we obtain the following equation by plugging in the first two equality constraints into the objective function,

$$
\begin{aligned}
f= & P\left(x^{A}+x^{B}\right)\left(x^{A}+x^{B}\right)-x^{A}\left(g\left(\frac{x^{A}}{I^{A} W^{A}}\right)+\frac{c I^{A}}{x^{A}}\right) \\
& -x^{B}\left(g\left(\frac{x^{B}}{I^{B} W^{B}}\right)+\frac{c I^{B}}{x^{B}}\right) \\
= & P\left(x^{A}+x^{B}\right)\left(x^{A}+x^{B}\right)-x^{A}\left(g\left(\frac{x^{A}}{I^{A} W^{A}}\right)+\frac{c}{W^{A}} \frac{I^{A} W^{A}}{x^{A}}\right) \\
= & -x^{B}\left(g\left(\frac{x^{B}}{I^{B} W^{B}}\right)+\frac{c}{W^{B}} \frac{I^{B} W^{B}}{x^{B}}\right)
\end{aligned}
$$

It follows that to maximize the profit, the monopolist needs to minimize $\Delta=x^{K}\left(g\left(\frac{x^{K}}{I^{K} W^{K}}\right)+\frac{c}{W^{K}} \frac{I^{K} W^{K}}{x^{K}}\right)$, where $K \in$ $\{A, B\}$.

\section{B. Proof for Section IV}

Lemma A.1: Assume the congestion costs function $g(y)$ is increasing, convex, then the following result holds,

$$
g\left(\frac{x_{1}}{\alpha I}\right) x_{1}+g\left(\frac{x_{2}}{\beta I}\right) x_{2} \geq g\left(\frac{x_{1}+x_{2}}{I}\right)\left(x_{1}+x_{2}\right),
$$

with $\alpha+\beta=1$.

To see this, we first let $f(x)=g(x / I) x$. It follows that $g\left(x_{1}^{A} / \alpha I\right) x_{1}^{A}=\alpha f\left(x_{1}^{A} / \alpha\right)$ and $g\left(x_{1}^{B} / \beta I\right) x_{1}^{B}=\beta f\left(x_{1}^{B} / \beta\right)$. Also it can be shown that $f(x)$ is convex in $x$ given the convexity and monotonicity of $g$. Hence, $\alpha f\left(x_{1}^{A} / \alpha\right)+\beta f\left(x_{1}^{B} / \beta\right) \geq$ $f\left(x_{1}^{A}+x_{1}^{B}\right)$. This completes the proof of the lemma.

\section{Proof for Theorem 4.2}

It has been shown that the monopoly equilibrium exists under the condition that $P\left(x^{*}\right) \leq \Delta$. To see the linear congestion satisfies this, it is sufficient to show $g\left(x^{*} / I_{M}\right) \leq$ $\Delta$, i.e., $x^{*} / I_{M} \leq \Delta$. In the monopoly equilibrium, the investment and number of customers have the property that $x_{M} / I_{M}=t^{*}$, where $t^{*}$ is the minimizer for $g(t)+c / t$. It follows that $t^{*}$ satisfies $g^{\prime}\left(t^{*}\right) t=c / t$, i.e., $g(t)=\Delta / 2$. Given that the monopolist invest in $I_{M}$ while serving $x_{M}$ customers, it follows that $g\left(x_{M} / I_{M}\right)=\Delta / 2$. Since $g(x / I)$ is linear, we have that $g\left(2 x_{M} / I_{M}\right)=\Delta$. Let $x_{\Delta}$ be the number of customers that satisfies $P\left(x_{\Delta}\right)=\Delta$. Thus to show $g\left(x^{*} / I_{M}\right) \leq \Delta$, it is sufficient to show that $g\left(x_{\Delta} / I_{M}\right)<\Delta$. In other words, $x_{M}>x_{\Delta} / 2$. We will show that this is true due to the optimization objective of the monopolist. By plugging in the constraints and taking derivative of the objective function, it follows that $P\left(x_{M}\right)+P\left(x_{M}\right)^{\prime} x_{M}=\Delta$. Given that $\Delta=P\left(x_{\Delta}\right)$, we have $P\left(x_{M}\right)+P\left(x_{M}\right)^{\prime} x_{M}=P\left(x_{\Delta}\right)$. Due to the concavity and monotonicity of the inverse demand function $P(x)$, it follows that $x_{M} \geq x_{\Delta}$, which establishes the needed inequality. 